\title{
INFLUENCE OF POST-CURE TREATMENTS ON HARDNESS AND MARGINAL ADAPTATION OF COMPOSITE RESIN INLAY RESTORATIONS: AN IN VITRO STUDY
}

\author{
Laiza Tatiana POSKUS', Antonio Marcelo Accetta LATEMPA², Maurício Alves CHAGAS³, Eduardo Moreira da SILVA', \\ Mariana Pareira da Silva LEAL ${ }^{4}$, José Guilherme Antunes GUIMARÃES ${ }^{1}$
}

\begin{abstract}
1-PhD, Assistant Professor, Department of Restorative Dentistry, Dental School, Federal Fluminense University, Niterói, RJ, Brazil. 2-DDS, Undergraduate student, Department of Restorative Dentistry, Dental School, Federal Fluminense University, Niterói, RJ, Brazil. 3- PhD, Department of Morphology, Biomedical Institute, Federal Fluminense University, Niterói, RJ, Brazil.

4- Graduate student, Department of Restorative Dentistry, Dental School, Federal Fluminense University, Niterói, RJ, Brazil.
\end{abstract}

Corresponding address: Laiza Tatiana Poskus - Faculdade de Odontologia - Universidade Federal Fluminense - Rua São Paulo, 30 - $3^{\circ}$ andar Valonguinho

Niterói - Rio de Janeiro - 24040-110 - Phone: 21 2629-9826/ 21 8136-8049 - e-mail: poskus@vm.uff.br

Received: November 19, 2008 - Modification: February 15, 2009 - Accepted: August 11, 2009

\begin{abstract}
O

bjectives: The purpose of this study was to evaluate the Vickers hardness number (VHN) and the in vitro marginal adaptation of inlay restorations of three hybrid composite resins (Filtek Z250, Opallis and Esthet-X) subjected to two post-cure treatments. Material and Methods: For the microhardness test, three different groups were prepared in accordance with the post-cure treatments: control group (only light cure for $40 \mathrm{~s}$ ), autoclave group (light cure for $40 \mathrm{~s}+$ autoclave for $15 \mathrm{~min}$ at $130^{\circ} \mathrm{C}$ ); and microwave group (light cure for $40 \mathrm{~s}+$ microwave for $3 \mathrm{~min}$ at $450 \mathrm{~W}$ ). To assess the marginal adaptation, the composite resin was inserted incrementally into a mesial-occlusal-distal cavity brass mold and each increment light-cured for $40 \mathrm{~s}$. A previous reading in micrometers was taken at the cervical wall, using a stereomicroscope magnifying glass equipped with a digital video camera and image-analysis software. Subsequently, the specimens were subjected to the post-cure treatments (autoclave and microwave) and a reading was taken again at the cervical wall. Data were compared using ANOVA for the hardness test, split-plot ANOVA for the adaptation assessment and Tukey's test for multiple comparisons. A significance level of 5\% was adopted for all analyses. Results: The post-cure treatments increased the hardness of conventional composites $(p<0.001)$ and the gap values of inlay restorations $(p<0.01)$. Filtek Z250 showed higher hardness $(p<0.001)$ and lower gap values than Opallis and Esthet-X $(p<0.05)$. Gap values did not exceed $90 \mu \mathrm{m}$ for any of the experimental conditions. Conclusion: The post-cure treatments increased the VHN and the gap values on the cervical floor of composite resin inlays. Moreover, Filtek Z250 showed the best results, with higher hardness and lower gap values.
\end{abstract}

Key words: Composite resins. Inlays. Cure. Hardness. Marginal adaptation.

\section{INTRODUCTION}

The great demand of patients for tooth-colored restorations or metal-free restorations and the improvements in the physical properties of composite resins have increased the indications of these materials for esthetic restorations. Nowadays, indirect composite resin inlay and onlay restorations are extensively used in esthetic treatments as a less expensive and less technique-sensitive alternative to ceramic crowns ${ }^{3}$. Moreover, the indirect technique improves the control of marginal adaptation, proximal contacts, anatomic form and polymerization shrinkage, compared to the direct composite resin technique ${ }^{30}$. Consequently, indirect composite resin restorations have shown less microleakage at the tooth-restoration interface than direct composite resins ${ }^{25}$.
The direct composite resin inlay/onlay technique was introduced to improve the adaptation in Class II cavities ${ }^{1}$, being less expensive than indirect ones and easily built up clinically. In this technique, the composite resin is first lightcured directly in the inlay cavity and then the inlay is removed from the cavity and post-cured. Extra-oral cure, which may occur under light, heat and/or pressure, increases the degree of conversion of dental composites, producing significant improvements in their mechanical and physical properties, such as hardness, elastic modulus, flexural strength, stiffness, hygroscopic expansion and solubility $10,18,20,27$. However, Peutzfeldt and Asmussen ${ }^{17}$ (1991) did not find these results for all composite resins evaluated in their study.

When direct inlays are fabricated, direct composite resins are used instead of ceromer materials, reducing the final 
treatment costs ${ }^{7}$. The mechanical properties of composites are comparable or superior to those of ceromers ${ }^{26}$. The higher degree of conversion caused by the secondary cure in the direct inlay technique can lead to greater polymerization shrinkage ${ }^{2}$ and, consequently, the marginal adaptation could be compromised ${ }^{28}$. Directly fabricated inlays have demonstrated good marginal integrity ${ }^{29}$ and are more accurate as regards gap formation than indirectly fabricated inlays ${ }^{16}$, particularly when the cervical margin is in dentin ${ }^{25}$.

After secondary cure, the inlay/onlay restoration is luted into place with composite resin luting materials. Therefore, the shrinkage is limited to the cement layer. However, the resin luting agents have some limitations, such as high shrinkage and low resistance to wear ${ }^{22}$. If the adaptation is poor, the resin cement layer will not be thin enough, and the longevity of the indirect restoration could be influenced ${ }^{19,22}$.

The purpose of this study was to evaluate the effects of two different post-cure treatments on the Vickers hardness number (VHN) and marginal adaptation of composite resin inlay restorations.

\section{MATERIAL AND METHODS}

The main characteristics of the three composites evaluated in this study (Esthet-X/Dentsply, Filtek Z250/3M and Opallis/FGM) are presented in Figure 1.

For the Vickers hardness test, two 2-mm-thick composite resin increments were inserted into a conical brass mold (3 and $4 \mathrm{~mm}$ diameter and $4 \mathrm{~mm}$ high), each one being lightcured for $40 \mathrm{~s}$ with a halogen light-curing unit (Optilux 501; Demetron/Kerr, Danbury, CT, USA, output $490 \mathrm{mw} / \mathrm{cm}^{2}$ ). Light unit irradiance was monitored using a radiometer (model 100, Demetron/Kerr). Fifteen specimens were made for each composite resin and divided into three experimental groups, in accordance with the post-cure treatments: control group (no post-cure treatment); autoclave group: the specimens were maintained during $15 \mathrm{~min}$ under $2.0 \mathrm{kgf} /$ $\mathrm{cm}^{2}$ and $130 \pm 1{ }^{\circ} \mathrm{C}$ in an autoclave (BIOS 19L; Dabi Atlante, Ribeirão Preto, SP, Brazil); microwave group: the specimens were treated for 3 min under medium power of $450 \mathrm{~W}$ in a domestic microwave oven (MB6544W, Samsung, Korea). The specimens were embedded in PVC tubes using epoxy resin (Trok-Dente, Prodens, RJ, Brazil) and were polished with 220-, 400-, 600- and 1200-grit abrasive papers and felt discs using diamond paste (Arotec, SP, Brazil) in a polishing machine (DPU-10, Struers, Copenhagen, Denmark). After $24 \mathrm{~h}$ of storage in distilled water, three indentations for VHN assessment (2003, Büehler, Lake Bluff, IL, USA) were performed in each specimen, under $50 \mathrm{~g}$ load and $15 \mathrm{~s}$ dwell time. The mean of the three measurements for each specimen was calculated and subjected to statistical analysis by two-way ANOVA and Tukey's test at 5\% significance level.

For marginal adaptation assessment, 2-mm-thick composite resin increments were inserted into a brass mold that simulated a mesial-occlusal-distal cavity. The dimensions and shape of the mold are shown in Figure 2. Each increment was light-cured for $40 \mathrm{~s}$ with the halogen light-curing unit (Optilux 501; Demetron/Kerr) and light unit irradiance was also monitored, as mentioned before. Ten specimens were made for each composite. After replacing the specimens in the brass mold, a monochrome charge-coupled device (CCD) video camera (TA-0123 Opton, Hexasystems, SP, Brazil) captured the images of the inlay restorations under a stereomicroscope magnifying glass (PZO, Metrimpex, Hungary) at $\times 10$ magnification. These images were transferred to an IBM-compatible personal computer for quantitative assessment in micrometers of marginal adaptation at four points (Figure 3), using Image1.36 image-analysis software (National Institute of Health, Bethesda, MD, USA). Two points were located at the cervical margin (mesial and distal) and two points at the internal angles (mesial-cervical-axial and distal-cervicalaxial). After removing the specimens from the mold, they were divided into two experimental groups, in accordance with the post-cure treatments: autoclave group and microwave group, which were described previously. The specimens were replaced again into the brass mold and the cervical adaptation was measured again, using the stereomicroscope, as mentioned above. The mean of the four measurements was calculated for each specimen. Data were

\begin{tabular}{|c|c|c|}
\hline Composite resin & Composition & Batch number \\
\hline $\begin{array}{l}\text { Filtek Z250 (3M ESPE, St. Paul, } \\
\text { MN, USA) }\end{array}$ & $\begin{array}{l}\text { Matrix: Bis-GMA, UDMA, Bis-EMA } \\
\text { Filler: } 0.01 \mu \mathrm{m}-3.5 \mu \mathrm{m} \text { with a mean particle size of } 0.6 \mu \mathrm{m} \\
(60 \mathrm{vol} \%)\end{array}$ & $5 R B$ \\
\hline $\begin{array}{l}\text { Esthet-X (Dentsply, Konstanz, } \\
\text { Germany) }\end{array}$ & $\begin{array}{l}\text { Matrix: Bis-GMA, Bis-EMA, TEGDMA } \\
\text { Filler: mean particle size bellow } 1 \mu \mathrm{m} \text { with nanofiller }(0.04 \\
\mu \mathrm{m})(60 \mathrm{vol} \%)\end{array}$ & 510311 \\
\hline $\begin{array}{c}\text { Opallis (FGM, Joinville, SC, } \\
\text { Brazil) }\end{array}$ & $\begin{array}{c}\text { Matrix: Bis-GMA, Bis-EMA, TEGDMA } \\
\text { Filler: } 40 \mathrm{~nm}-3.0 \mu \mathrm{m} \text { with a mean particle size of } 0.5 \mu \mathrm{m} \\
(57 \mathrm{vol} \%)\end{array}$ & 31006 \\
\hline
\end{tabular}

Bis-GMA: Bisphenol A-diglycidyl ether dimethacrylate; UDMA: Urethane dimethacrylate; Bis-EMA: bisphenol-Apolyethylene glycol diether dimethacrylate; TEGDMA: Tri[ethylene glycol] dimethacrylate.

FIGURE 1- Composition of the composite resins evaluated in the study 


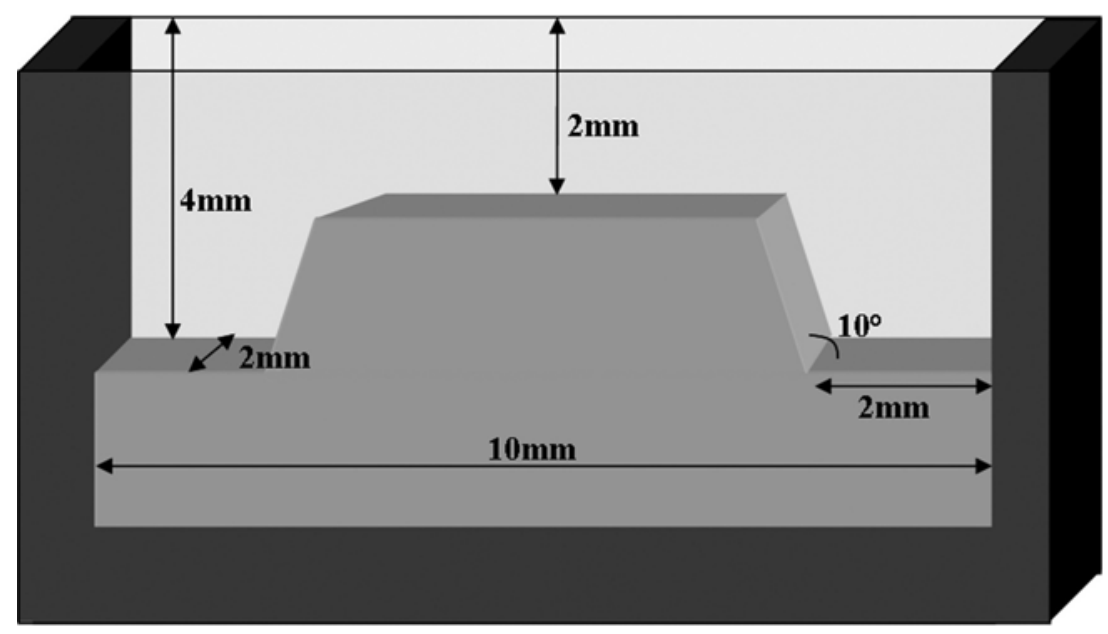

FIGURE 2- Dimensions and shape of the standard inlay cavity

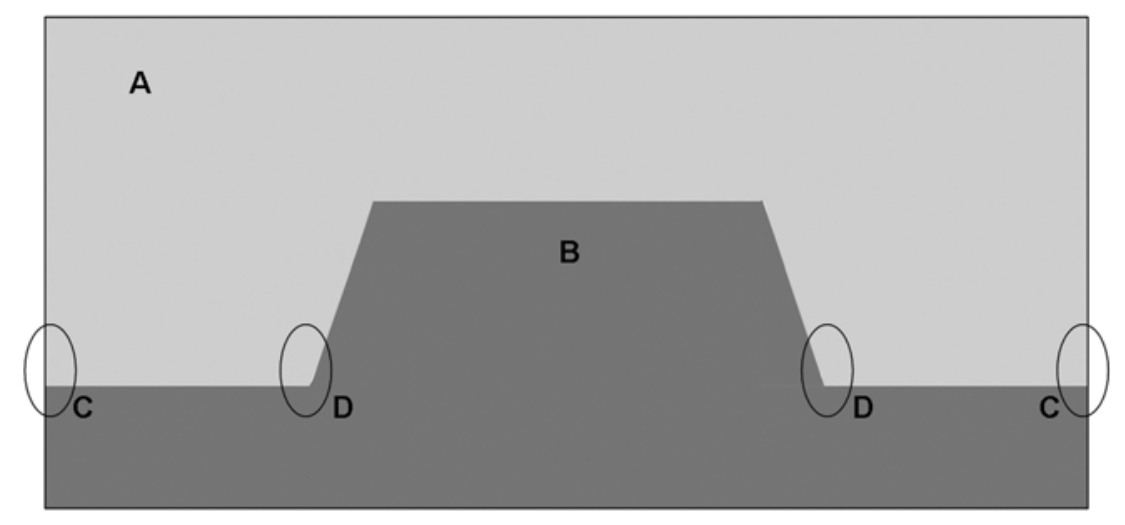

FIGURE 3- Gap measurement points: (A) composite resin; (B) brass mold; (C) cervical margin (mesial and distal); (D) internal angles (cervical-axial, mesial and distal)

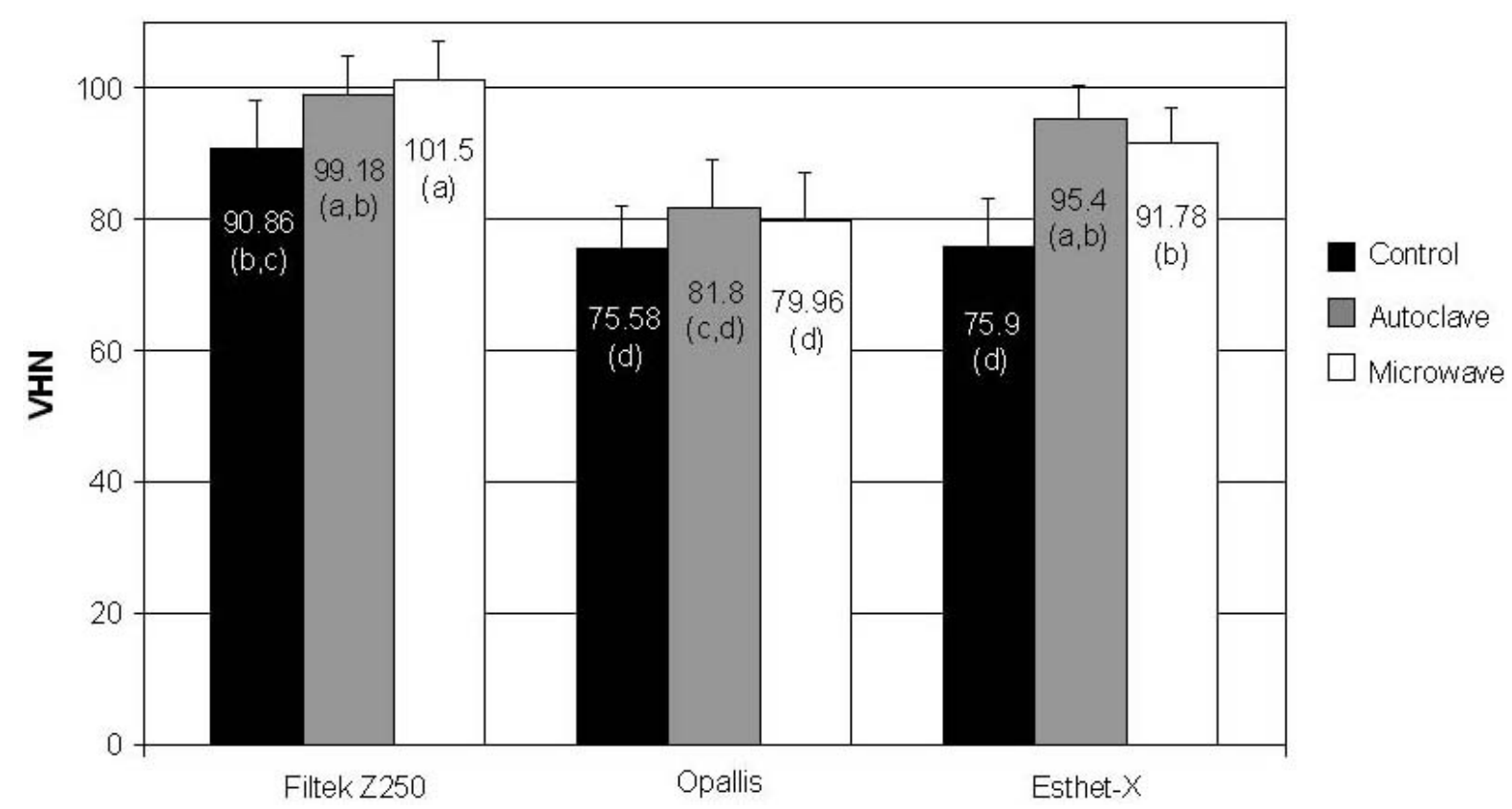

FIGURE 4- Mean and standard deviation of the VHN for each material and post-cure treatment. Different lower case letters show statistically significant diferences 
TABLE 1- Mean, standard deviation, minimum and maximum of gap values $(\mu \mathrm{m})$

\begin{tabular}{|c|c|c|c|c|}
\hline \multirow[t]{2}{*}{ Composite resin } & \multicolumn{4}{|c|}{ Post-cure treatment } \\
\hline & Before & After & Minimum & Maximum \\
\hline Filtek Z250 & $13.02[11.64]^{\mathrm{a}, \mathrm{A}}$ & $19.69[15.87]^{\mathrm{b}, \mathrm{A}}$ & 0 & 61.36 \\
\hline Opallis & $23.95[11.19]^{a, B}$ & $32.68[13.31]^{\mathrm{b}, \mathrm{B}}$ & 5.48 & 64.85 \\
\hline Esthet-X & $22.56[15.50]^{a, B}$ & $32.74[18.07]^{\mathrm{b}, \mathrm{B}}$ & 0 & 81.46 \\
\hline
\end{tabular}

Different lowercase letters in the rows and uppercase letter in the columns indicate statistically significant difference.

analyzed by split-plot ANOVA and Tukey's test at 5\% significance level.

\section{RESULTS}

Figure 4 and Table 1 present the means and standard deviation of the VHN and gap width values as a function of the post-cure treatments.

Regarding Vickers hardness analysis, ANOVA showed that the VHN was significantly affected by the composite materials $(\mathrm{p}<0.001)$ and the post-cure treatments $(\mathrm{p}<0.001)$. The highest VHN was found for Filtek Z250, followed by the Esthet-X and Opallis. Moreover, all post-cure treatments increased the VHN when compared to the control group, though without statistically significant difference $(p>0.05)$ between them. For Opallis, neither autoclave nor microwave was able to increase the VHN (Figure 4). For Esthet-X, both post-cure treatments, increased the VHN $(\mathrm{p}<0.01)$, whereas for Filtek Z250, only the microwave group showed higher VHN, when compared with the control group $(\mathrm{p}<0.01)$.

Regarding the adaptation assessment, split-plot ANOVA showed that the composite materials and the post-cure treatments influenced the gap width values. The lowest width gap values (Table 1) were found for the inlay restorations that were not subjected to any post-cure treatment $(\mathrm{p}<0.001)$. No statistically significant difference was found between the two experimental groups (autoclave or microwave). Filtek Z250 showed significantly $(\mathrm{p}<0.05)$ lower gap values (Table 1) than Opallis and Esthet-X.

\section{DISCUSSION}

It is well known that, at room temperature, the conversion of composite resin $\mathrm{C}=\mathrm{C}$ bonds is not complete ${ }^{23}$, ranging between 48 and $60 \%{ }^{8,24}$. For indirect resin restorations, additional cure procedures have been used to increase the degree of conversion, improving the mechanical properties ${ }^{18}$. With secondary cure the chain vibration amplitude is increased allowing free radicals and methacrylate groups to collide and establish covalent links, increasing the degree of conversion ${ }^{21}$. It has been shown that the hardness of composites correlates well with degree of conversion ${ }^{6}$. In the present study, the post-cure treatments improved the VHN. This result is in agreement with those of other authors ${ }^{10,27}$.

If the VHN had increased, the degree of conversion might be higher, leading to greater cure shrinkage and, consequently, poor marginal adaptation. In the present study, the gap values on the cervical floor increased with the postcure treatments, also indicating a higher degree of conversion. Gap values under $90 \mu \mathrm{m}$ were found for all experimental conditions (Table 1). Schmalz, et al. ${ }^{22}$ found that with a luting space of up to $100 \mu \mathrm{m}$, the width of the luting space has no influence on the marginal integrity.

The adaptation was evaluated directly in the brass mold, mimicking the clinical conditions for the direct composite resin inlay/onlay technique, since no impression was taken. When the marginal and overall adaptation of inlays was observed by using the silicon replica technique, gap values over $100 \mu \mathrm{m}$ were found ${ }^{15}$. For this technique, the impression material, the stone and the silicone fitness-checking could have some influence on the gap formation between cavity walls and inlays. The use of silicon impression has never been considered adequate to exactly replicate the behavior of dental cement in the clinical situation ${ }^{19}$.

Although Opallis and Esthet-X had shown similar VHN for the control group, they showed lower VHN than Filtek Z250, corroborating finding of Correr, et al. ${ }^{4}$ (2005) for Esthet-X composite. As their manufacturers propose a longer working time, it can be speculated that a diminished content of photoinitiators might be present, leading to a lower degree of conversion and a lower VHN with the cure protocol used in the present study. Therefore, with the secondary cure by microwave or autoclave, the network would have higher mobility, improving the cure and, consequently, the VHN. This factor was statistically significant for Esthet-X, which showed an increase of $20.9 \%$ to $25 \%$ with both post-cure treatments (microwave and autoclave), but not for Opallis. However, the VHN for Opallis increased about $8.2 \%$ for the autoclave group and $5.8 \%$ for the microwave group, indicating an improvement in VHN with the post-cure treatments. In a previous study ${ }^{5}$, after 1-week storage, an additional light exposure also increased the VHN for Esthet$\mathrm{X}$.

For Filtek Z250, VHN also tended to increase (9.8\%) when the samples were autoclaved and there was a significant difference ( $11.7 \%$ increase) between the control and microwave groups (Figure 3). Other authors have suggested that the faster cure rate of the Z100 might be caused by its high content of photoinitiators ${ }^{9,12}$. Although 
the technical profiles of 3M ESPE do not specify the content of photoinitiators in their composites and neither the aforementioned studies nor the present study made this evaluation in the tested materials, it can be hypothesize that, since Filtek Z250 was developed from Filtek Z100 (Technical Product Profile, 3M ESPE), a higher level of photoinitiators might be present in both materials, explaining the higher degree of conversion and, consequently, the higher VHN shown for Filtek Z250 (Figure 3). Supporting this statement, Hofmann, et al. ${ }^{13}$ (2002) attributed the faster cure reaction of Filtek $\mathrm{Z} 250$ to the high efficiency of the photoactivation system of this material.

Still following the same thought, if the degree of conversion before the post-cure treatments was very high for Filtek Z250, the mobility of the network would be more difficult ${ }^{11}$ during the post-cure treatments, leading to a lower extent of post-cure (9.8-11.7\%), when compared to Esthet$\mathrm{X}$. These speculations could also explain the best adaptation on the cervical floor for Z250 $(\mathrm{p}<0.05)$. If its conversion with the post-cure treatment were lower, its polymerization shrinkage would also be lower, leading to the formation of smaller gaps. The very low content of TEGDMA in Filtek $\mathrm{Z} 250^{8}$, a smaller molecular size monomer, might also explain the best adaptation for this material, as it could be expected a lower shrinkage than that for Opallis and Esthet-X.

Since there was no significant difference between autoclave and microwave groups, it can be emphasized that both treatments were efficient for enhancing the VHN, which is in agreement with Soares, et al. ${ }^{26}(2005)$ Furthermore, after the secondary cure, the gap values were higher but similar for both groups. Others studies also verified an improvement in the degree of conversion when light was used in conjunction with heat and pressure ${ }^{8,14,24}$.

Within the limitations of this in vitro study, the postcure treatments for the tested composites were efficient in increasing the $\mathrm{VHN}$, without significantly compromising the marginal adaptation of the inlay restorations, as gap values did not exceed $90 \mu \mathrm{m}$. Therefore, in wide Class II cavities, the lower cost of the direct composite resin inlay/onlay, as well as being less time-consuming technique, would be an advantage. Additional clinical studies are necessary to analyze the cervical adaptation, marginal leakage and wear resistance, confirming the efficiency of this technique.

\section{CONCLUSIONS}

Based on the methodology of the present study, it may be conclude that: 1 . The post-cure treatment with autoclave or microwave increased the VHN and the gap values on the gingival floor of composite resin inlays; 2. Filtek Z250 showed the highest VHN and the best marginal adaptation of all composites.

\section{ACKNOWLEDGEMENTS}

The authors would like to thank the PIBIC/CNPq for finacial support.

\section{REFERENCES}

1- Blankenau RJ, Kelsey WP $3^{\text {rd }}$, Cavel WT. A direct posterior restorative resin inlay technique. Quintessence Int Dent Dig. 1984;15(5):515-6.

2- Calheiros FC, Braga R, Kawano Y, Ballester RY. Relationship between contraction stress and degree of conversion in restorative composites. Dent Mater. 2004;20(10):939-46.

3- Chalifoux PR. Restoration of endodontically treated teeth: review, classification and post design. Pract Periodontics Aesthet Dent. 1998;109(2):247-54.

4- Correr AB, Sinhoreti MA, Correr L Sobrinho, Tango RN, Schneider LF, Consani S. Effect of increase of energy density on Knoop hardness of dental composites light-cured by conventional QTH, LED and xenon plasma arc. Braz Dent J. 2005;16(3):218-24.

5- De Jong LC, Opdam NJ, Bronkhorst EM, Roeters JJ, Wolke JG, Geitenbeek B. The effectiveness of different polymerization protocols for class II composite resin restorations. J Dent. 2007;35(6):513-20.

6- DeWald JP, Ferracane JL. A comparison of four modes of evaluating depth of cure light activated composites. J Dent Res. 1987;66(3):727-30.

7- Duke ES. The introduction of a new class of composite resins ceromers. Compend Contin Educat Dent. 1999;20(3):246-7.

8- Emami N, Soderholm KJ. How light irradiance and curing time affect monomer conversion in light-cured resin composites. Eur J Oral Sci. 2003;111(6):536-42.

9- Ernst CP, Kürschner R, Rippin G, Willershausen B. Stress reduction in resin-based composites cured with a two-step light-curing unit. Am J Dent. 2000;13(2):69-72.

10- Ferracane JL, Condon, JR. Post-cure heat treatments for composites: properties and fractography. Dent Mater. 1992;8(5):290-5.

11- Halvorson RH, Erickson RL, Davidson CL. Energy dependent polymerization of resin-based composite. Dent Mater. 2002;18(6):463-9.

12- Hasegawa T, Itoh K, Yukitani W, Wakumoto S, Hisamitsu H. Effects of soft-start irradiation on the depth of cure and marginal adaptation to dentin. Oper Dent. 2001;26(4):389-95.

13- Hofmann N, Hugo B, Klaiber B. Effect of irradiation type (LED or QTH) on photo-activated composite shrinkage strain kinects, temperature rise and hardness. Eur J Oral Sci. 2002;110(6):471-9.

14- Kakaboura A, Rahiotis C, Zinelis S, Al-Dhamadi YA, Silikas N, Watts DC. In vitro characterization of two laboratory-processed resin composites. Dent Mater. 2003;19(5):393-8.

15- Karakaya S, Sengun A, Ozer F. Evaluation of internal adaptation in ceramic and composite resin inlays by silicon replica technique. J Oral Rehabil. 2005;32(6):448-53.

16- Peutzfeldt A, Asmussen E. A comparison of accuracy in seating and gap formation for three inlay/onlay techniques. Oper Dent. 1990;15(4):129-35.

17- Peutzfeldt A, Asmussen E. Mechanical properties of three composite resins for the inlay/onlay technique. J Prosthet Dent. 1991;66(3):322-4. 
18- Peutzfeldt A, Asmussen E. The effect of postcuring on quantity of remaining double bonds, mechanical properties and in vitro wear of two resin composites. J Dent. 2000;28(6):447-52.

19- Qualtrough AJ, Piddock V, Kypreou V. A comparison of two in vitro methods for assessing the fitting accuracy of composite inlays. Br Dent $\mathrm{J}$. $1993 ; 174(12): 450-4$.

20- Reinhardt JW, Boyer DB, Stephens NH. Effects of secondary curing on indirect posterior composite resins. Oper Dent. 1994;19(6):217-20.

21- Rueggeberg FA, Ergle JW, Lockwood PE. Effect of photoinitiator level on properties of a light-cured and post-cure heated model resin system. Dent Mater 1997;13(6):360-4.

22- Schmalz G, Federlin M, Reich E. Effect of dimension of luting space and luting composite on marginal adaptation of a class II ceramic inlay. $\mathrm{J}$ Prosthet Dent. 1995;73(4):392-9.

23- Sideridou I, Tserki V, Papanastasiou G. Effect of chemical structure on degree of conversion in light-cured dimethacrylate-based dental resins. Biomaterials. 2002;23(8):1819-29.

24- Silva GRD, Simamoto PC Jr, Mota AS. Mechanical properties of light- curing composites polymerized with different laboratory photocuring units. Dent Mater J. 2007;26(2):217-23

25- Soares CJ, Celiberto L, Dechichi P, Fonseca RB, Martins LRM. Marginal integrity and microleakage of direct and indirect composite inlays - SEM and stereomicroscopic evaluation. Braz Oral Res. 2005;19(4):295 301.

26- Soares CJ, Pizi EC, Fonseca RB, Martins LRM. Mechanical properties of light cured composites polymerized with several additional post-curing methods. Oper Dent. 2005;30(3):389-94.

27- Takeshige F, Kinomoto Y, Torii M. Additional heat-curing of lightcured composite resin for inlay restoration. J Osaka Univ Dent Sch. 1995;35:59-66

28- Tarle Z, Meniga A, Knezevic A, Sutalo J, Ristic M, Pichler G. Composite conversion and temperature rise using a conventional, plasma arc, and experimental blue LED curing unit. J Oral Rehabil. 2002;29(7):662-7.

29- Van DijKen JW, Hörstedt P. Marginal breakdown of 5-year-old direct composite inlays. J Dent. 1996;24(6):389-94.

30- Wassel RW, Walls AW, McCabe JF. Direct composite inlays versus conventional composite restorations: three-year clinical results. Br Dent J. 1995;179(9):343-9. 